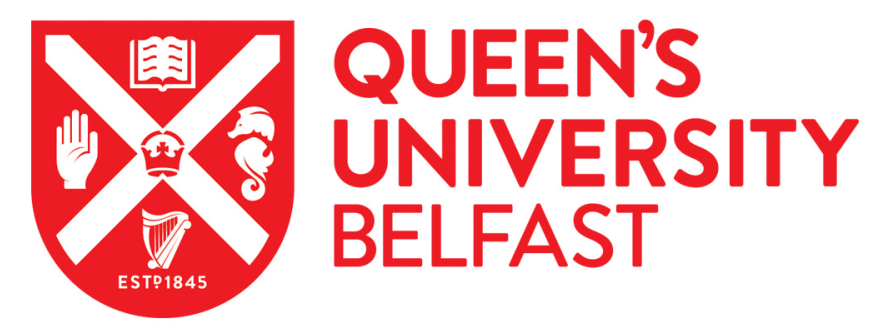

\title{
An Antennas and Propagation Approach to Improving Physical Layer Performance in Wireless Body Area Networks
}

Conway, G. A., Cotton, S. L., \& Scanlon, W. G. (2009). An Antennas and Propagation Approach to Improving Physical Layer Performance in Wireless Body Area Networks. IEEE Journal on Selected Areas in Communications, 27(1), 27-36. [4740883]. https://doi.org/10.1109/JSAC.2009.090104

Published in:

IEEE Journal on Selected Areas in Communications

Document Version:

Early version, also known as pre-print

Queen's University Belfast - Research Portal:

Link to publication record in Queen's University Belfast Research Portal

Publisher rights

() 2009 IEEE.

\section{General rights}

Copyright for the publications made accessible via the Queen's University Belfast Research Portal is retained by the author(s) and / or other copyright owners and it is a condition of accessing these publications that users recognise and abide by the legal requirements associated with these rights.

Take down policy

The Research Portal is Queen's institutional repository that provides access to Queen's research output. Every effort has been made to ensure that content in the Research Portal does not infringe any person's rights, or applicable UK laws. If you discover content in the Research Portal that you believe breaches copyright or violates any law, please contact openaccess@qub.ac.uk. 


\title{
An Antennas and Propagation Approach to Improving Physical Layer Performance in Wireless Body Area Networks
}

\author{
Gareth A. Conway, Simon L. Cotton, Member, IEEE, and William G. Scanlon, Member, IEEE
}

\begin{abstract}
A combined antennas and propagation study has been undertaken with a view to directly improving link conditions for wireless body area networks. Using tissue-equivalent numerical and experimental phantoms representative of muscle tissue at $2.45 \mathrm{GHz}$, we show that the node to node $\left|S_{21}\right|$ path gain performance of a new wearable integrated antenna (WIA) is up to $9 \mathrm{~dB}$ better than a conventional compact Printed-F antenna, both of which are suitable for integration with wireless node circuitry. Overall, the WIA performed extremely well with a measured radiation efficiency of $38 \%$ and an impedance bandwidth of $24 \%$. Further benefits were also obtained using spatial diversity, with the WIA providing up to $7.7 \mathrm{~dB}$ of diversity gain for maximal ratio combining. The results also show that correlation was lower for a multipath environment leading to higher diversity gain. Furthermore, a diversity implementation with the new antenna gave up to $18 \mathrm{~dB}$ better performance in terms of mean power level and there was a significant improvement in level crossing rates and average fade durations when moving from a singlebranch to a two-branch diversity system.
\end{abstract}

Index Terms-Body area network; wearable antennas; channel characterization; diversity; on-body channels.

\section{INTRODUCTION}

$\mathbf{T}$ HE LAST decade has witnessed a growing interest in wearable sensing and computing devices. This has led to the concept of a body area network (BAN), with standardization work currently underway by the IEEE 802.15 .6 committee. The topology of a wireless BAN may consist of several nodes placed at various locations on the body (Fig. 1), each composed of sensor circuitry, embedded signal processing and an RF transceiver module. Potentially, these BANs could offer significant benefits in commercial, military and medical applications. For example, a BAN may be used for data exchange between a bodyworn media device and wireless headset [1], [2], or for a network of wearable sensors configured to remotely monitor vital signs for managing and supervising the health status of patients [3]. Clearly, these systems are required to be compact, lightweight, robust, unobtrusive to the user and ideally conformal to the body surface, yet they must maintain high performance in terms of reliability and efficiency. Each of these factors present considerable

Manuscript received 31 January 2008; revised 28 September 2008. This work was supported by the UK Department of Employment and Learning and partially funded by the UK Engineering and Physical Science Research Council (EP/D053749/1).

The authors are with the School of Electronics, Electrical Engineering and Computer Science, Queen's University, Belfast, BT3 9DT, UK. (e-mail: gconway03@ecit.qub.ac.uk; simon.cotton@qub.ac.uk; w.scanlon@qub.ac.uk)

Digital Object Identifier 10.1109/JSAC.2009.090104.

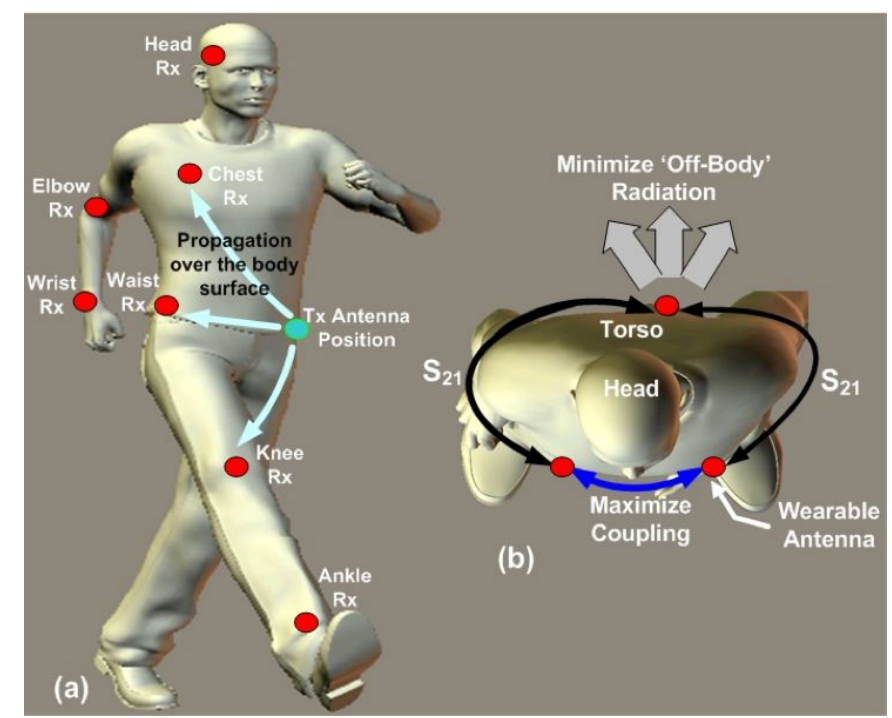

Fig. 1. Concept body area network: a) example node positions, b) illustration of general antenna radiation requirements for coupling between nodes.

design challenges at various layers in the protocol stack, with issues related to system integration [4], device miniaturization [5], low power design [6], and wireless security [7] currently being actively addressed.

Improvements in physical layer performance may be attained through the proficient design of transceiver modules for BAN nodes. Two key areas where enhancements can be realized are antennas designed specifically for bodyworn use and the exploitation of the unique propagation characteristics found in BANs. It is widely accepted that antenna performance is significantly affected by close proximity to the human body [8]-[12]. For example, radiation pattern distortion, reduced radiation efficiency and changes in antenna input impedance are commonly reported [10]-[12]. The antenna must also be suitable for integration with RF and baseband circuitry in BAN nodes. However, to date, there has been little published research on wearable antennas specifically designed for integration within BAN systems. In general, printed antennas offer a low-profile antenna solution for wearable electronic systems with favorable characteristics such as physical robustness, low-cost, ease of manufacture and can easily be integrated with electronic circuitry. Microstrip patches are suitable for wearable applications [9], [12] and can offer reduced body tissue losses if their groundplanes are sufficiently large and 
placed adjacent to the body surface. However, when integrated with circuitry, the patch design dictates that the circuit is placed either below the groundplane (next to the body surface) or to one side of the antenna on an extended groundplane, potentially degrading the system performance through reduced over-the-body-surface (on-body) propagation or mutual coupling, respectively.

The mobile nature of the human body requires the BAN to operate in diverse environments that, in terms of propagation, can be broadly classified as either anechoic or multipath. Ideally, for reliable and efficient communication between wearable devices, their performance should be independent of the environment in which they are used. The on-body channel between two nodes in a BAN is also largely affected by user movement. Natural physical body movements such as breathing, walking and running will vary the propagation across the surface of the body, changing the BAN channel characteristics, causing fading in received signal levels [13] which, depending on severity and frequency, may cause momentary loss of the on-body link. Bodyworn diversity is one potential method for increasing the mean received signal level and improving fading characteristics for the on-body channels found in BANs, although it has received relatively little attention to-date [13], [14]. Receiver diversity aims to provide a higher signal-to-noise ratio (SNR) by combining multiple space [15], time [16], frequency [17] or polarization [18] separated signal branch contributions.

In this work, we focus on the improvements which may be made to the physical layer, firstly, by introducing a novel wearable integrated antenna (WIA) for use in BAN applications and secondly, by performing a characterization of spatially separated dual-antenna systems to mitigate against signal fading in BANs. Simulated and measured path gain performance for the WIA in close proximity to a lossy medium representing body tissues at $2.45 \mathrm{GHz}$ is reported. Comparisons of these parameters with a conventional Printed-F antenna mounted on the same substrate are given such that the merits the new antenna can be firmly established. Both the Printed-F and WIA antennas were also evaluated in terms of simulated and measured return loss, bandwidth and radiation efficiency for on-body communication. Other major contributions of this work include a study of mobile on-body diversity systems operating in anechoic and dense multipath conditions. Signal parameters such as cross-correlation, difference in mean signal level between receiver branches and diversity gain for dualbranch systems with integrated antennas are reported.

The antennas and propagation issues and requirements relating to body area networks are discussed in Section II of this paper. Section III details the design and construction of the antenna prototypes, and a comprehensive study of their on-body performance presented. A diversity characterization of spatially separated dual-antenna systems for BANs is performed in Section IV. Section V then proceeds to demonstrate the benefits of diversity using first and second order statistics for an on-body link with the user mobile in a strong multipath environment. The report concludes with a summary of the findings and suggestions for further work.

\section{Wireless Body AREA Network CONCEPT AND Physical Layer Challenges}

On-body channels exist where there is a need for communication between devices located on, or extremely close to, a user's body [Fig.1(a)]. Generally, the intended application dictates the precise location of devices which, in turn, will have a major effect on system performance. Path length and visibility (line of sight, LOS; or non line of sight, NLOS) tend to govern the mean received signal power, while placing the nodes on a limb will increase the body's influence on the channel characteristics, especially for a mobile user.

\section{A. BAN Antenna Requirements}

The antenna presents a significant problem in the miniaturization of integrated BAN nodes as performance is directly related to physical size, involving a trade-off between design parameters such as mass, efficiency, bandwidth and radiation characteristics. Furthermore, these effects will vary between different antennas, groundplane size, separation distances and near-field proximity coupling to the body. The radiation characteristics of the antenna have a significant influence on signal propagation over the body surface. At UHF and higher frequencies, penetration through the body is significantly reduced and the main mechanism for propagation around the body is via a creeping wave that follows the dielectric-air interface at the body surface [19]. In anechoic environments, on-body devices without LOS will communicate via this creeping wave, while in a multipath environment there may be propagating waves diffracted or reflected from nearby objects such as building partitions and furniture. Consequently, on-body wearable applications require a low-profile antenna which directs maximum radiation tangential to the body surface, minimizing off-body radiation, maximizing $\left(\left|S_{21}\right|\right)$ path gain between bodyworn devices [Fig. 1(b)].

\section{B. On-Body Diversity Receiver}

For a diversity receiver to be effective the mean signal level in each of the receiver branches should be comparable and the fading suitably uncorrelated. This may be a particular problem for bodyworn spatial diversity systems where the small size of nodes may provide insufficient antenna spacing to ensure uncorrelated fading in each receiver branch, potentially reducing the maximum achievable diversity gain. Furthermore, in anechoic-like environments (e.g., outdoors or larger indoor areas) the received signal for on-body channels may be formed from two or more unequal length paths around the perimeter of the human body. This may also reduce diversity gain because of the increased power imbalance at the input of the diversity receiver. It is therefore essential that the magnitude of correlation and power imbalance within each branch be established as this will directly impact the effectiveness of implementing diversity within wireless BAN nodes.

\section{WEARABLE INTEGRATED ANTENNA}

The new WIA antenna [Fig. 2(a)] incorporates innovation in terms of both feed structures and radiation characteristics. 

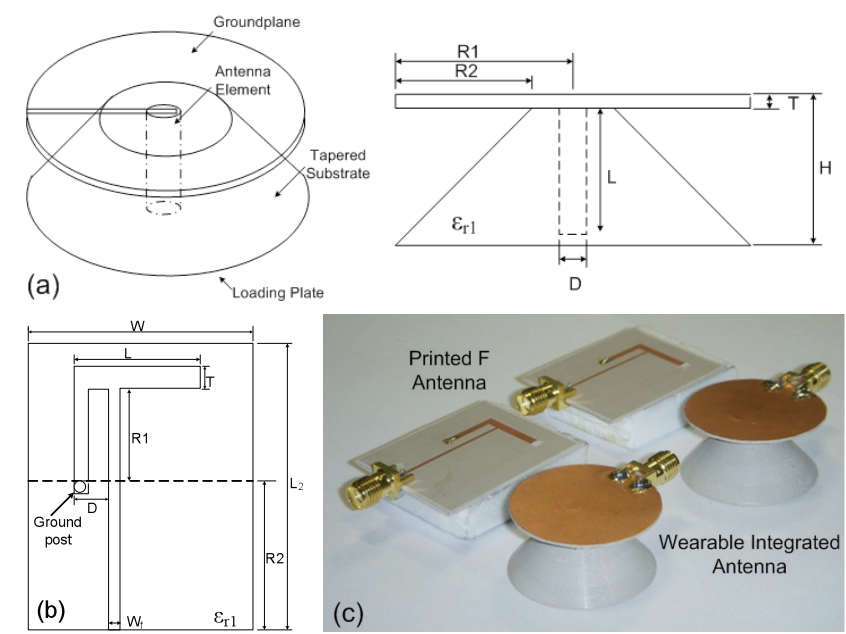

Fig. 2. Antenna geometry: (a) WIA, (b) Printed-F, (c) prototypes.

It was designed with its groundplane furthest away from the body surface so that RF and baseband circuitry could be placed adjacent to or above the groundplane (on the opposite side to the radiating element) without degrading the antenna performance. The reduction in mutual coupling between the electronics and the antenna also removes the need to redesign the antenna following circuit design changes. The WIA antenna radiates with maximum gain tangential to the tissue surface, thus supporting the propagating creeping wave mode (with the electric field normal to the tissue surface). Antennas which support this surface wave are known to give improved path gain performance for on-body links [9].

Using Finite Difference Time Domain (FDTD) simulations, the WIA and Printed-F antennas were optimized for tissue mounted operation at the ISM (Industrial Scientific and Medical) frequency allocation of $2.4-2.5 \mathrm{GHz}$ with a nominal resonant frequency of $2.45 \mathrm{GHz}$ compatible with the IEEE 802.15.1 [20] and IEEE 802.15.4 [21] standards. The WIA consisted of a $3 \mathrm{~mm}$ diameter copper element embedded in a tapered Taconic CER 10 dielectric substrate $\left(\epsilon_{r 1}=10.2\right)$. The element, fed by a microstrip line, is also loaded with a copper plate spaced $0.5 \mathrm{~mm}$ from the top of the radiating element. The loading plate is closest to the tissue surface and helps reduce direct coupling between the tissue and element, minimizing variations in impedance with antenna-body separation distance and improving on-body performance due to lower tissue losses. FDTD analysis showed that tapering the dielectric substrate improved antenna performance in terms of both return loss and on-body propagation, and it also reduced the mass of the antenna. The Printed-F antenna [Fig. 2(b)] was constructed on $0.6 \mathrm{~mm}$ thick CER 10 substrate. Key dimensions for each antenna are given in Table I.

\section{A. Simulation and Measurement Setup}

To investigate antenna $\left|S_{21}\right|$ path gain performance via creeping waves the antennas were placed on opposite sides of a specially shaped tissue-equivalent phantom (Fig. 3), removing the LOS propagation path. The permittivity and conductivity of the phantom were chosen to represent muscle tissue at $2.45 \mathrm{GHz}\left(\epsilon_{r}=53.58, \sigma=1.81 \mathrm{Sm}^{-1}\right)$ [22]. A phantom

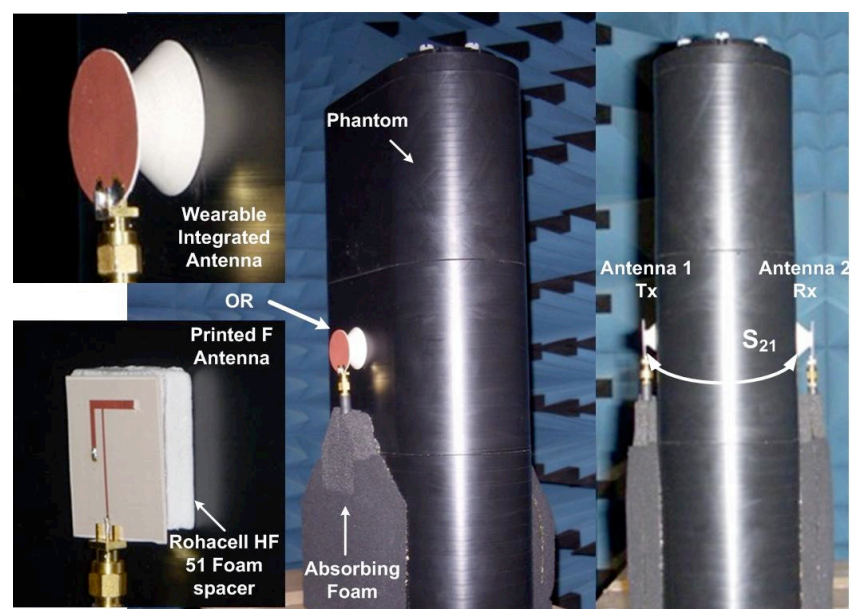

Fig. 3. Tissue phantom and $\left|S_{21}\right|$ measurement set-up in anechoic chamber.

thickness of $100 \mathrm{~mm}$ was chosen to effectively eliminate signal penetration through the structure and hence isolate the creeping wave propagating mode. A numerical anechoic modeling environment was achieved using absorbing boundaries in the FDTD computational domain, while physical measurements were made in the quiet zone of an anechoic chamber. The rationale for the chosen test set up was as follows: (i) measurements on phantoms minimize uncontrolled factors such as body movement (e.g., respiration) and changes in body posture allowing repeatable test environment to evaluate and compare the performance of each antenna; (ii) in anechoic environments with NLOS paths, waves propagating over a lossy curved surface are diffracted and attenuated, effectively reducing the received signal power thus, providing a worst case (in terms of signal propagation) antenna test environment. The phantom was physically realized using a Nylon $66\left(\epsilon_{r}=3.4, \sigma=0.04\right.$ $\mathrm{Sm}^{-1}$ ) shell with a wall thickness of $2 \mathrm{~mm}$. The phantom was filled with a lossy dielectric solution, used to represent human muscle tissue at $2.45 \mathrm{GHz}$, details can be found in [9].

The $\left|S_{21}\right|$ measurements were made using a ZVB8 vector network analyzer ${ }^{1}$ (VNA). Rohacell HF 51 foam $\left(\epsilon_{r}=1.07\right)$ was used to space the Printed-F antenna $9 \mathrm{~mm}$ above the phantom to represent realistic conditions in integrated devices, since operation directly against the user's body would be extremely inefficient. The WIA was placed on the surface of the phantom with no spacing. Emerson and Cuming Eccosorb LS-16 and LS-24 microwave absorbing foam with an insertion loss of $1.5 \mathrm{~dB} / \mathrm{cm}$ and $11 \mathrm{~dB} / \mathrm{cm}$, respectively were used to minimize spurious radiation from, and coupling between, the coaxial test cables. All simulated results in this work were obtained with the SEMCAD- $\mathrm{X}^{2}$ FDTD electromagnetic numerical modeling platform.

\section{B. Return Loss and Bandwidth Characteristics}

Fig. 4 shows the measured and simulated return loss results for each antenna. The WIA had a measured impedance bandwidth $\left(\left|S_{11}\right| \leq-10 \mathrm{~dB}\right)$ of $24 \%$, and the Printed-F antenna had a bandwidth of $6 \%$, both more than sufficient

\footnotetext{
${ }^{1}$ Rohde \& Schwarz Inc., Columbia MD.

${ }^{2}$ Schmid \& Partner Engineering AG, Zürich, Switzerland.
} 
TABLE I

Antenna Mass and Principal Dimensions for Operation on Tissue Phantom at $2.45 \mathrm{GHz}$

\begin{tabular}{c|c|ccccccccc}
\hline \multirow{2}{*}{ Antenna } & \multirow{2}{*}{ Prototype mass $(\mathrm{g})$} & \multicolumn{10}{|c}{ Dimensions (mm), refer to Fig. 2 } \\
\cline { 3 - 10 } & & $L$ & $L_{2}$ & $W$ & $H$ & $T$ & $R 1$ & $R 2$ & $W_{f}$ & $D$ \\
\hline WIA & 5.0 & 11 & - & - & 12.5 & 0.5 & 15 & 10 & 0.8 & 3.0 \\
Printed-F & 12.0 & 15 & 37 & 30 & - & 2.0 & 10 & 20 & 0.8 & 4.5 \\
\hline
\end{tabular}

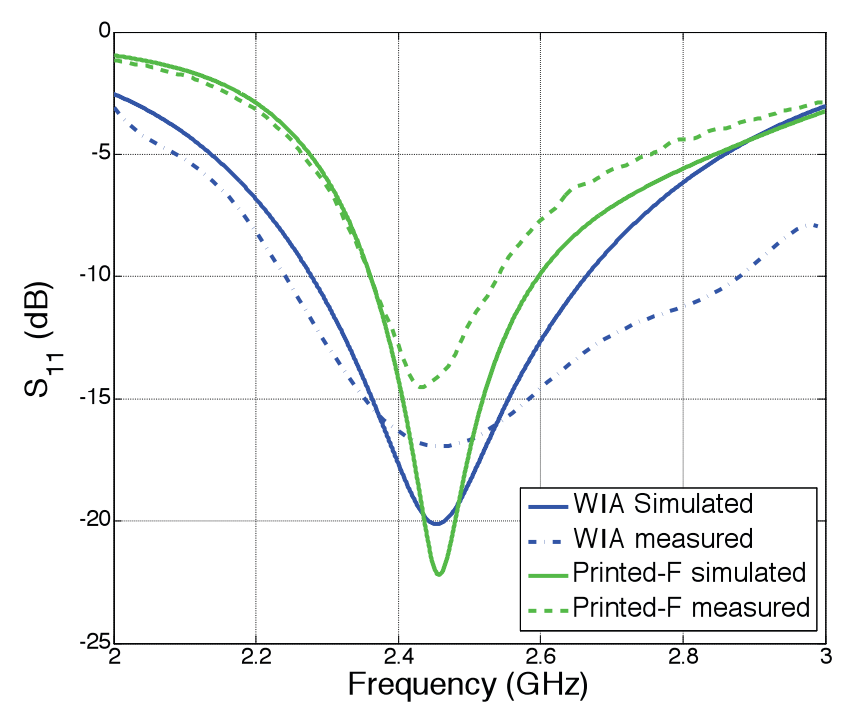

Fig. 4. Simulated and measured antenna return loss (on phantom).

for the requirements of the $2.45 \mathrm{GHz}$ ISM band (3.4\%). Excess bandwidth is advantageous in wearable antenna design as it can compensate for small fluctuations in the resonant frequency, caused by variations in antenna body-coupling due to changes in antenna separation distances, particularly when the user is mobile. It should be noted that because of its loading plate structure, the WIA was not particularly sensitive to antenna-body separation distance. Measurements showed that it was matched across the entire ISM band for separations of up to $10 \mathrm{~mm}$ off the phantom. Overall, the simulated $\left|S_{11}\right|$ results were in good agreement with measurements.

\section{On-Body Coupling in Anechoic Chamber}

Measurements in the anechoic chamber (Fig. 5) show that the WIA had $8 \mathrm{~dB}$ greater path gain than the Printed-F antenna with a peak $\left|S_{21}\right|$ of $-42 \mathrm{~dB}$ at $2.45 \mathrm{GHz}$. This significant increase in path gain was achieved as the WIA radiates tangentially to the tissue surface, therefore supporting the dominant propagation mode in this anechoic environment. There was good agreement between the peak $\left|S_{21}\right|$ measurements and FDTD simulation with a discrepancy of less than $1 \mathrm{~dB}$ at $2.45 \mathrm{GHz}$.

A reverberation chamber was used to measure the total radiation efficiency of each antenna when mounted on the surface of the phantom. The measured and FDTD simulated radiation efficiency of the Printed-F antenna at $2.45 \mathrm{GHz}$ was $10.5 \%$ and $14 \%$, respectively, compared to $38 \%$ (measured) and $45 \%$ (simulated) for the WIA. The simulated efficiency results were in good agreement with measurements with less than $1 \mathrm{~dB}$ discrepancy. The Printed-F antenna was less efficient as the radiating element was placed planar to the body surface

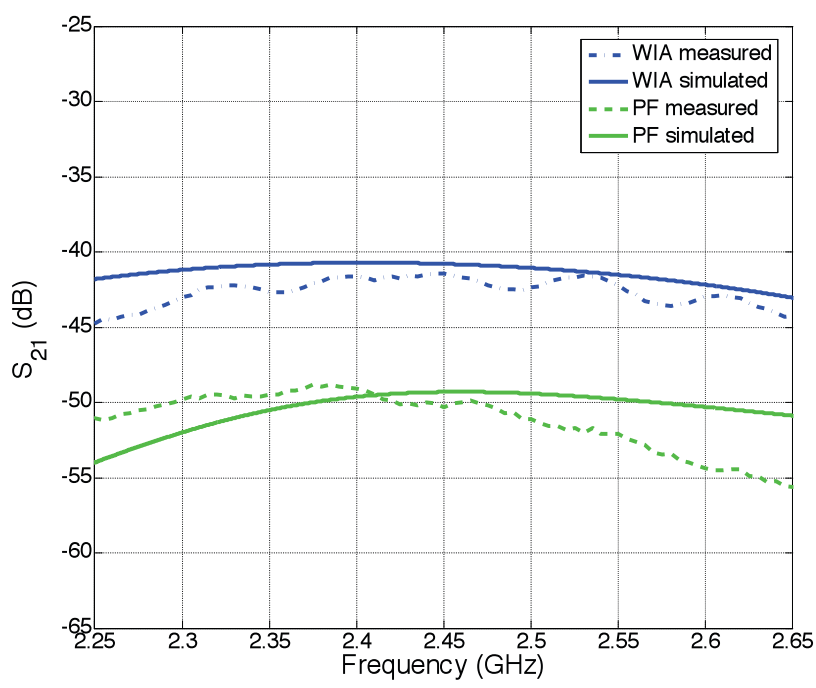

Fig. 5. Simulated and measured $\left|S_{21}\right|$ path gain for on-body antennas.

and the parallel $E$-field is shorted by the conductive body tissues.

\section{On-Body Coupling in Reverberation Chamber}

The performance of antennas designed for on-body channels are usually primarily evaluated in an anechoic operating environment. Nonetheless, it is also appropriate to determine their performance under multipath conditions since the presence of off-body paths may significantly improve on-body links for some antenna types. This was investigated by considering the on-body performance of the antennas in the controlled multipath environment of a reverberation chamber. The chamber used was a shielded room of size $2.4 \times 2.4 \times 2.4 \mathrm{~m}$, and it was equipped with fixed metallic baffles, two mechanical plate stirrers, polarization stirring and platform stirring. The reverberation chamber generates a repeatable strong multipath environment with fading characteristics which closely follow the Rayleigh distribution. This was confirmed using maximum likelihood estimation on the full set of channel gain results for the WIA antenna on-phantom link. The analysis showed that the Nakagami- $m$ distribution gave the best fit, with a fading parameter $m=1.14$ (the Nakagami distribution is equivalent to Rayleigh when $m=1$ ).

The measurements were conducted in the range 2.25$2.65 \mathrm{GHz}$ and the resulting $\left|S_{21}\right|$ path gain performance is shown in Fig. 6. Both of the antennas were subjected to identical multipath conditions by following the same computercontrolled set of stirrer and platform positions. In comparison to the results obtained in the anechoic environment, peak $\left|S_{21}\right|$ values were approximately $7 \mathrm{~dB}$ higher in the reverberation chamber due to the strong multipath environment. Therefore, 


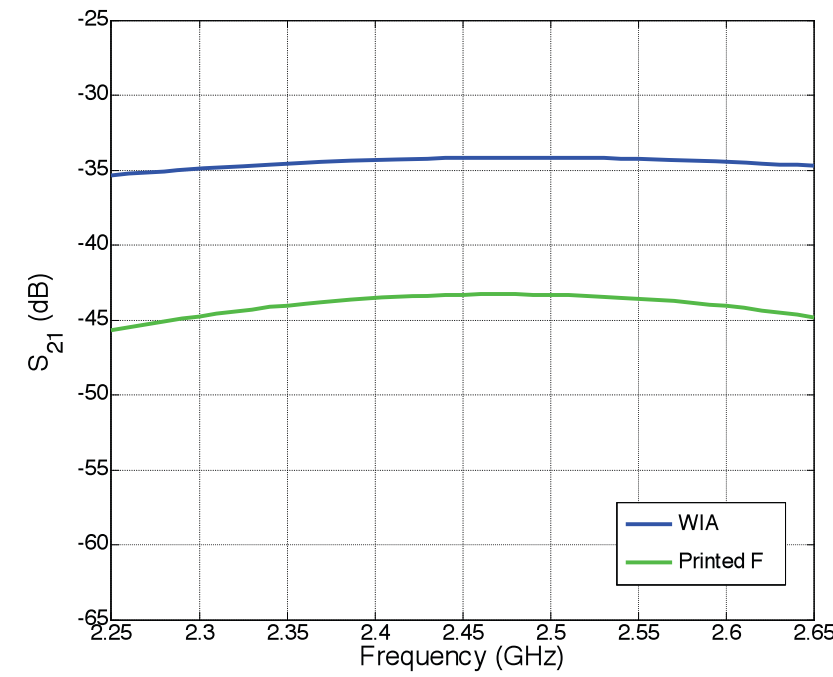

Fig. 6. Measured $\left|S_{21}\right|$ path gain for on-body antennas in a reverberation chamber.

in practice the performance of the antennas will vary between that the anechoic chamber results and the reverberation chamber results, depending on the environment in which they are used. The WIA also performed better in the multipath environment, with $9 \mathrm{~dB}$ more path gain than the Printed-F antenna. Hence, regardless of the propagation environment, the WIA offers a significant improvement in on-body performance compared to the Printed-F. This extra system gain may be used to provide additional advantage in wireless BAN networks, e.g., in terms of increased battery life, improved on-body range, or less stringent transceiver specifications.

\section{Diversity Characterization for Mobile BANs}

The experiments performed in this study were carefully chosen to evaluate the full range of performance of spatial diversity systems for use in BANs. Two separate measurement environments were chosen which were considered to be representative of the minimum (anechoic chamber) and maximum (reverberation chamber) environmental multipath conditions likely to be experienced by everyday BAN users. Based on earlier on-body measurement campaigns at $868 \mathrm{MHz}$ [13] and $2.45 \mathrm{GHz}$ [23], it is known that implementing receiver diversity is most beneficial if the transmitter and receiver are situated on opposite sides of the user's body. Therefore, the transmit antenna used in this study (representative of a BAN node) was fixed to one side of the user's waist and the receive antennas on the side of the head, front chest, waist, knee, ankle, wrist, elbow and back chest all situated on the opposite side of the user's body, as illustrated in Fig. 1(a). This set up also ensured that NLOS conditions existed between the transmitter and receiver. Two horizontally separated dualantenna diversity systems consisting of the Printed-F or WIA antennas discussed in Section III were investigated. In both cases the two antennas were aligned side by side (Fig. 7) such that the main radiating elements of the dual-antenna node were separated by a straight-line separation distance of $30 \mathrm{~mm}$ $(0.245 \lambda$, overall array dimensions $60 \times 30 \mathrm{~mm})$ for the WIA

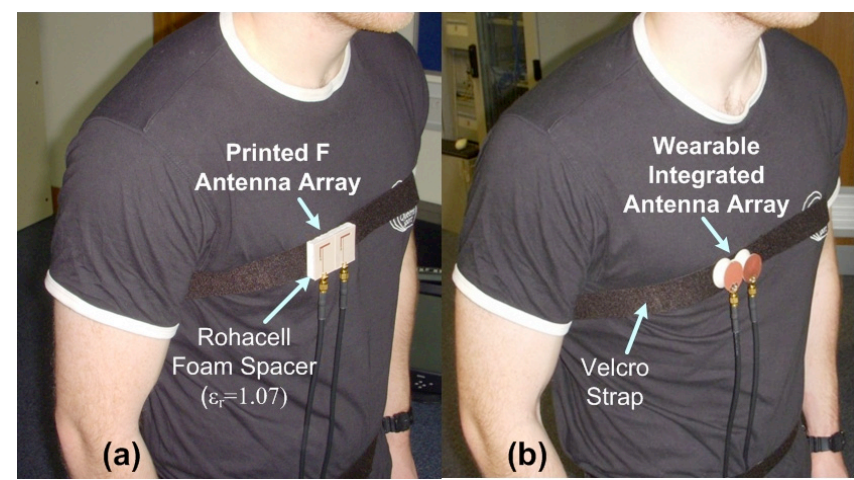

Fig. 7. Bodyworn dual antenna diversity setup: (a) Printed-F (b) WIA.

and $15 \mathrm{~mm}(0.123 \lambda$, overall array dimensions $60 \times 37 \mathrm{~mm})$ for the Printed-F.

For each experiment, the transmit antenna was attached to port 1 of the ZVB-8 VNA and was identical to that used at the receiver array (ports 2 and 3), i.e., all three antennas were either WIA or Printed-F. Since the VNA had independent receivers and reflectometers it was configured to record simultaneous synchronized measurements of $\left|S_{21}\right|$ and $\left|S_{31}\right|$ at $5 \mathrm{~ms}$ intervals. The RF output of port 1 was set to a level of $0 \mathrm{dBm}$ at $2.45 \mathrm{GHz}$ and calibrated low-loss coaxial cables were used for all connections. All of the diversity measurements involved the user walking in a normal fashion with an approximate maximum speed of $1 \mathrm{~ms}^{-1}$ within the anechoic chamber and reverberation chamber environments described earlier. Each of the individual, pre-combined received signal envelopes analyzed in this study consisted of 10000 samples, giving a cumulative total of 320000 samples for both antennas over the two environments. This was also the sample set size used for all subsequent diversity and statistical analyses.

\section{A. Correlation Between Diversity Branches}

The cross-correlation coefficient (CCC), $\rho_{c}$, between the fading envelopes $r_{1}$ (branch 1) and $r_{2}$ (branch 2) consisting of $N$ samples may be represented by [15]

$$
\rho_{c}=\frac{\sum_{i=1}^{N}\left[r_{1}(i)-\bar{r}_{1}\right]\left[r_{2}(i)-\bar{r}_{2}\right]}{\sqrt{\sum_{i=1}^{N}\left[r_{1}(i)-\bar{r}_{1}\right]^{2}} \sqrt{\sum_{i=1}^{N}\left[r_{2}(i)-\bar{r}_{2}\right]^{2}}},
$$

where $i$ is the instantaneous sample value, and $\bar{r}_{1}$ and $\bar{r}_{2}$ are the respective means of the signal envelopes. Turkmani [24] has shown that diversity gain is a function of branch correlation and power imbalance, with the gain tending to increase as the magnitude of these two parameters decrease. It is well known that for diversity systems which rely on spatially separated antennas, the correlation between the branch envelopes is a function of the spacing between the antennas, with correlation generally decreasing as the separation distance increases [25]. This is not usually an issue in non-bodyworn system design as antenna separation can just be increased until a suitable performance is attained. However, in BAN applications the antennas will most likely be very close to each other due to the necessarily compact design of transceiver nodes. Therefore, in this study the antennas were situated as close together as was physically possible. 
As well as antenna spacing, the signal correlation between branches in diversity arrays is also dependent upon a number of other factors; the first of these is mutual coupling between antenna elements. This was determined by measuring $\left|S_{21}\right|$ between the two array elements at $2.45 \mathrm{GHz}$ when placed upon the user's chest as shown in Fig. 7. Coupling was found be greatest for the WIA $(-15.4 \mathrm{~dB})$, despite this diversity array having a greater separation between the radiating elements than the Printed-F $(-17.2 \mathrm{~dB})$. Another important factor affecting correlation is the effect of the human body upon the antenna radiation pattern [10], [11] which, in mobile BAN applications, may be expected to further reduce correlation. However, the effect of the body may not be entirely beneficial, since radiation pattern distortion may lead to increased power imbalance between receiver branches. Finally, the level of multipath interference will play an important role in determining branch correlation in BANs. This will be particularly prominent within anechoic-like conditions where, because of the reduced multipath contribution from the local surroundings, diversity systems will have to depend on diffuse signal contributions caused by the multipath associated with the body itself to aid in the de-correlation of signal branches.

Table II gives $\rho_{c}$ for the fading envelopes received by the bodyworn diversity systems. As expected, due to the reduced level of environmental multipath, estimated CCCs for both the Printed-F and WIA antennas were much greater in the anechoic chamber than in the reverberation chamber. For both antennas in this environment, in most cases ( 6 out of the 8 systems) $\rho_{c}$ was less than 0.7 , below which it is generally accepted that, for balanced power within all signal branches, diversity combining will provide worthwhile gain [26]. Overall, the correlation between signal branches in the WIA based receivers was considerably lower than those obtained for the Printed-F, which may have been due to a combination of the improved antenna on-body propagation with the new antenna (Section III.C.) or, to a lesser extent, the increased spacing between radiating elements. In the strong multipath conditions of the reverberation chamber, $\rho_{c}$ was always less than 0.15 for the WIA. Correlation for the Printed-F was also generally low, with the exception of the waist mounted array $\left(\rho_{c}=0.61\right)$.

\section{B. Difference in Mean Signal Level and Diversity Gain}

Power imbalance between diversity branches can have a significant effect on the overall achievable diversity gain. To assess this, the mean signal level experienced by each of the spatially separated antennas was calculated from their raw signal voltage envelope and used to compute the difference in mean signal level (DIMSL) between branches. For both antenna types, a dramatic difference between the DIMSL was observed when moving from anechoic conditions to the highly multipath conditions of the reverberation chamber. Table II shows that for the Printed-F antenna, the DIMSL ranged from $0.88 \mathrm{~dB}$ (front chest) to $9.19 \mathrm{~dB}$ (knee) while the user was mobile in the anechoic chamber. This range in DIMSL values was significantly reduced when the measurements were repeated in the reverberation chamber ranging between $0.03 \mathrm{~dB}$ for the elbow to $3.82 \mathrm{~dB}$ for the waist position. This trend was repeated for the WIA which further benefited from much lower DIMSLs when compared to the Printed-F. From these results it may be deduced that environmental multipath will play an important role in improving the signal characteristics of diversity systems used in BANs through reduced envelope correlation and decreased DIMSL between signal branches.

A number of different combining schemes may be used to improve the output SNR within a diversity receiver. Some commonly encountered techniques are: selection combination (SC) where the signal with the highest SNR is selected; equal gain combining (EGC) in which the signal received on all branches is equally weighted, cophased (pre-detection only) and summed; and maximal ratio combining (MRC) where branch signals are weighted according their SNR, cophased (pre-detection only) and summed. Of these schemes, EGC is of particular interest as it provides comparable performance to MRC but at reduced complexity in terms of receiver implementation. The equations used to simulate SC, EGC and MRC combining of the raw received signal envelopes in post processing are given in equations (2)-(4) of [13], respectively. Diversity gain results were calculated from the combined envelopes according to [24] where an empirical definition of diversity gain is given as the difference in signal level of the branch with the highest mean and the of the output of the diversity combiner for a given probability or signal reliability. All diversity gain calculations in this paper were made at a signal reliability of $90 \%$ (a cumulative probability of 0.1 ).

In agreement with the estimated CCCs and DIMSLs, diversity gain was found to significantly increase for both antenna types when the user switched from the anechoic to the highly multipath conditions of the reverberation chamber (Table II). The WIA-based diversity system provided very little gain in the anechoic chamber, with the exception of the head and ankle located receivers. A plausible explanation for this is that as this antenna was designed to maximize coupling over the body surface, it is likely that the mean received signal level of the direct (shortest) on-body path will be much greater than waves arriving from other on-body directions. This concept can be seen in Fig. 1(a) where any signal contribution arriving along the back of the body at the receiver on the user's waist will be greatly reduced when compared to the EM contribution propagating across the front of the body. Since the head and ankle positions are at the opposite extremities of the body in relation to the transmit antenna in this study, it is unlikely that either of these positions will receive a continuous creeping wave [19] contribution during user movement. Instead, even in anechoic-like conditions, these positions will be dominated by body-surface multipath, reducing correlation and DIMSL between branches and improving the diversity gain obtained. Furthermore, although the WIA-based receiver generally provided lower diversity gains in the anechoic chamber than the Printed-F diversity system, it did provide much better average received signal level. To further show the advantage of using the WIA, we provide another measure of diversity performance which may be used to compare different diversity receivers, the mean diversity power level (MDPL). The MDPL is calculated by obtaining the mean power level of the received signal envelope after diversity combining. The choice of combining scheme is arbitrary but must be kept consistent when comparing between 


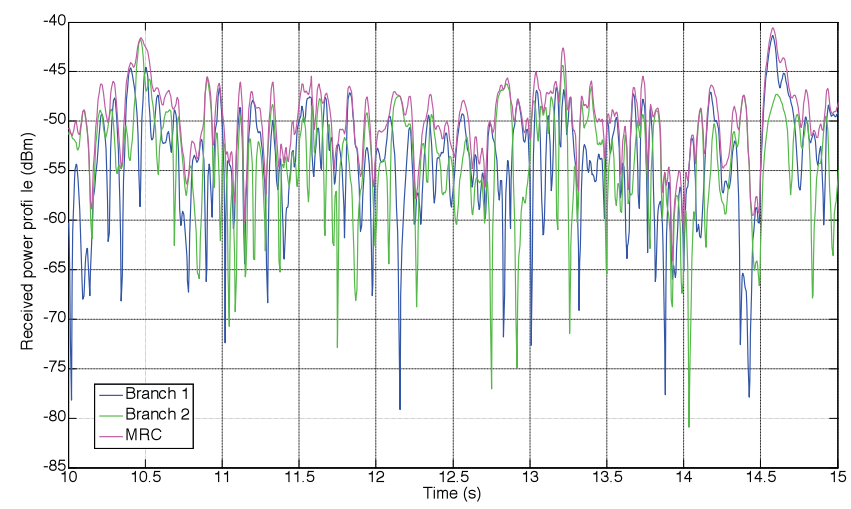

Fig. 8. Extract from received signal envelope time series between 10-15 s for a mobile user with a head-mounted WIA diversity configuration in the reverberation chamber.

different diversity systems. As shown in Table II, despite providing lower diversity gains in the anechoic chamber, the WIA arrangement significantly outperformed the Printed-F in terms of MDPL (for MRC combining) by almost $18 \mathrm{~dB}$ for the front chest receiver position. This is significantly higher than the $9 \mathrm{~dB}$ advantage given by the WIA in the single (nondiversity) antenna case shown in Section III.C.

\section{A Selection of Fading Statistics For DUAL-ANTENNA BAN NODES}

Alongside the improvement in the received signal in terms of diversity gain, further evidence of the benefits which may be obtained by using more than one signal branch in bodyworn applications may also be provided by analyzing the amplitude statistics of the combined signal envelope. In the following examples, we focus on channel characteristics for the dualantenna nodes operating in the reverberation chamber where the full benefits of a diversity configuration may be observed. In particular, we examine the waist to head on-body channel which occurs when wireless headsets are used in conjunction with waist mounted cellular devices.

In some previous studies, the global mean [23], or local mean [27] if the mean signal slowly varies over time, are removed to facilitate the extraction of small-scale fading characteristics. However, to allow direct comparison between diversity combining schemes the results presented here were un-normalized, calculated using the raw signal envelope. Fig. 8 shows a $5 \mathrm{~s}$ sample of the received power profile for both branches and the MRC combiner output for the head mounted diversity receiver. The improvement in signal characteristics are significant with all fades at lower signal levels $(<-75 \mathrm{dBm})$ completely eliminated. Further evidence of signal improvement may be obtained by examining the cumulative distribution functions (CDFs) before and after combining (Fig. 9). Also shown in Fig. 9 are the theoretical CDFs for SC [28], EGC [29] and MRC [28] combiners operating in Nakagami fading conditions. The Nakagami- $m$ distribution has been used in the analysis of diversity statistics for a number of decades [13], [30]. It has proven popular due to its ease of manipulation and its ability to approximate the Rice pdf [31] for $m>1$, reduce to the Rayleigh pdf $(m=1)$ and describe fading conditions which are worse

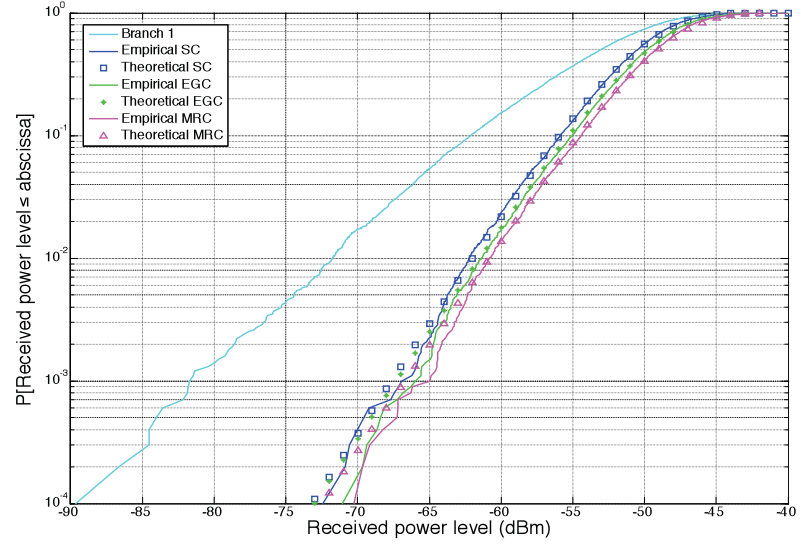

Fig. 9. Empirical and theoretical CDFs showing the improvement in channel characteristics when moving from a single antenna receiver using the branch with the highest mean level to dual-branch SC, EGC and MRC combiners. MLE parameters for theoretical plots are: $m_{S C}=0.904, \Omega_{S C}=0.367$, $m_{E G C}=0.877, \Omega_{E G C}=0.372, m_{M R C}=0.868, \Omega_{M R C}=0.377$.

than Rayleigh $(m<1)$. All distributional parameter estimates were obtained using maximum likelihood estimation (MLE) which was performed on a $95 \%$ confidence interval by minimizing the negative log-likelihood function over a bounded parameter space using the constrained minimization algorithm, fmincon(.), available in the optimization toolbox of Matlab. For all three combining schemes, the theoretical plots are shown to provide excellent agreement. Interestingly, all three estimates of the Nakagami- $m$ parameter (i.e., $m_{S C}=0.904$, $m_{E G C}=0.877$ and $\left.m_{M R C}=0.868\right)$ were $<1$ suggesting that channel conditions experienced by these receivers are worse than those for an SC, EGG or MRC combiner operating in Rayleigh fading conditions. It should be noted that, although obtained from the combined signal envelope, the Nakagami parameter estimates given here do not constitute the $m$ parameter for the envelope obtained at the output of the diversity combiner but rather, assuming i.i.d. fading, the perceived channel conditions for each receiver branch.

Two second order statistics which are of great importance in the design of mobile radio systems and analysis of their performance [23] are the level crossing rate (LCR), which is the number of times a signal crosses a threshold level in a positive (or negative) going direction, and the average fade duration (AFD), which is the total amount of time spent below the same threshold level. Fig. 10 shows the LCRs calculated from -90 to $-40 \mathrm{dBm}$ in steps of $0.5 \mathrm{~dB}$. Only the branch with the highest mean is shown alongside the LCR plots for each of the three combining schemes. The improvement in LCR when moving from a one branch receiver to a dualbranch one is substantial. For example, in branch 1, the received power profile crosses the $-60 \mathrm{dBm}$ power level with a frequency of $8 \mathrm{~Hz}$ which, even for the poorest performing combining technique (SC), is reduced to $2 \mathrm{~Hz}$ or below. Fig. 10 also shows that, as expected, the MRC combiner performed best, although only marginally better than EGC. Also shown in Fig. 10 is the theoretical LCR $\left(N_{R}\right)$ for a selection combining scheme with $M$ diversity branches operating in Nakagami 


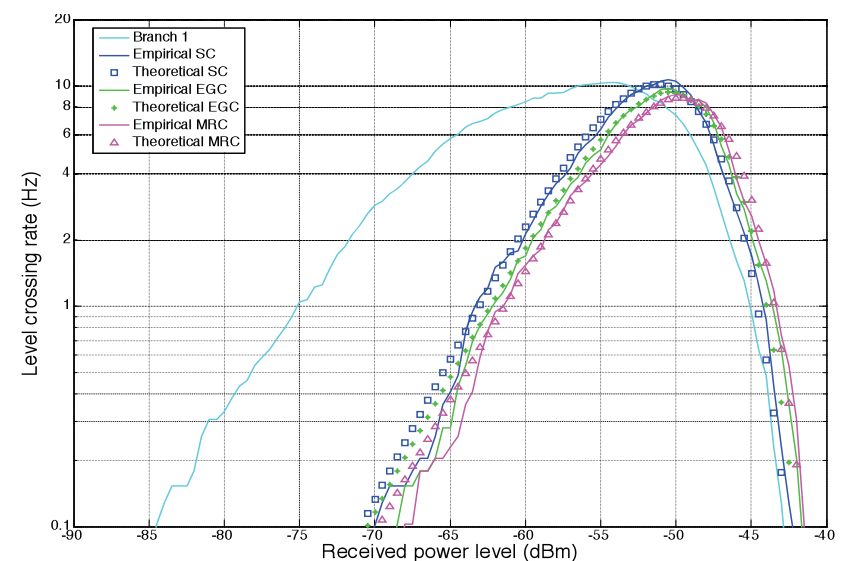

Fig. 10. Empirical and theoretical LCRs showing the improvement in channel characteristics when moving from a single antenna receiver using the branch with the highest mean level to dual-branch SC, EGC and MRC combiners. MLE parameters for theoretical plots are given in caption of Fig. 9.

fading conditions, calculated using [28].

$$
N_{R}=\frac{\sqrt{2 \pi} M \Gamma^{M-1}\left(m, m \rho^{2}\right) f_{m}}{\Gamma^{M}(m)}\left(m \rho^{2}\right)^{m-0.5} \exp \left(-m \rho^{2}\right),
$$

where $m$ is the Nakagami fading parameter, $\Omega$ the mean power, $\rho$ is the normalized threshold level $(R / \sqrt{\Omega})$ and $f_{m}$ is the maximum Doppler frequency. $\Gamma(a)$ and $\Gamma(a, b)$ denote the complete and incomplete Gamma functions, respectively. The LCR for MRC in similar fading conditions is given by [32]

$$
N_{R}=\frac{\sqrt{2 \pi} f_{m}}{\Gamma(m M)}\left(m \rho^{2}\right)^{m M-0.5} \exp \left(-m \rho^{2}\right) .
$$

No closed-form expression exists for the solution of the $M$ branch Nakagami EGC LCR. In [29], Iskander has expressed the LCR for a dual-branch EGC combiner with i.i.d. branch envelopes as

$$
\begin{array}{r}
N_{R}=\frac{\sqrt{2 \pi} B\left(2 m, \frac{1}{2}\right) f_{m}}{[\Gamma(m)]^{2} 2^{2 m-2}}\left(\frac{m}{\Omega} r^{2}\right)^{2 m-0.5} \exp \left(-2 \frac{m}{\Omega} r^{2}\right) \\
\times \Phi\left(2 m, 2 m+\frac{1}{2}, \frac{m}{\Omega} r^{2}\right) .
\end{array}
$$

where $B(a, b)$ is the beta function and $\Phi(a, b, c)$ is the confluent hypergeometric function. For all three LCR diversity plots, the theoretical equations are shown to provide a good description of the empirical data. Interestingly, the maximum Doppler frequencies of the theoretical equations which most closely match the measured data had $f_{m S C}=8.20 \mathrm{~Hz}$, $f_{m E G C}=8.57 \mathrm{~Hz}, f_{m M R C}=8.56 \mathrm{~Hz}$ which corresponds well with the theoretical maximum Doppler frequency for an approximate user speed of $1 \mathrm{~ms}^{-1}\left(f_{m}=8.2 \mathrm{~Hz}\right.$ at $\left.2.45 \mathrm{GHz}\right)$. Although not shown, a significant improvement in AFD when moving from a single to dual branch on-body receiver was also observed. For all three combining schemes, fades were well below the branch with the highest mean level. For example, at the $-55 \mathrm{dBm}$ power level, the average duration of fading below this level was $30 \mathrm{~ms}$ for SC with EGC and MRC further improving on this figure, compared to $40 \mathrm{~ms}$ for the single branch.

\section{CONCLUSION}

Energy conservation and link reliability are of particular concern within BAN applications where it is desirable to communicate across the human body with the minimum number of hops and at the lowest possible transmit power level. We have shown that the physical layer performance of a wireless BAN can be significantly improved using innovative antenna design and bodyworn antenna diversity. In comparison to a conventional integrated antenna, gains of up to $9 \mathrm{~dB}$ can be realized in BANs by using antennas such as the WIA that support a surface wave propagation mode. Further improvements may also be possible with more directional antenna designs which have greater gain tangential to the body surface. The WIA itself may be further optimized in terms of height and mass for specific applications where the impedance bandwidth requirements are lower. This may be achieved by embedding the radiating element in a substrate with higher dielectric constant or with the addition of a secondary plate connected to the end of the radiating element (top-loading) to increase its electrical length, significantly reducing the dielectric volume and hence mass of the antenna. Nonetheless, even in its current implementation, the WIA itself is a significant step forward, especially considering that it provides additional gain but with no drawback in terms of detuning, impedance bandwidth or radiation efficiency.

A diversity characterization for two different types of dual-branch bodyworn systems based on integrated antennas, including the WIA, has shown that cross-correlation and difference in mean signal levels between diversity branches is highly dependent upon local surroundings. For anechoic environmental conditions, cross-correlation was always found to greater for both the Printed-F and WIA spatial diversity systems compared to the dense multipath conditions of the reverberation chamber. Furthermore, the increased multipath signal contribution in the reverberation chamber environment acted to reduce DIMSLs compared to the anechoic chamber. In general, the WIA performed marginally better than the Printed-F in terms of diversity gain in the reverberation chamber but the anechoic chamber results were more dependent on receiver position. However, it was shown that, for a direct like-for-like comparison, the WIA offered up to $18 \mathrm{~dB}$ better performance in terms of mean diversity power level in anechoic conditions and almost $6 \mathrm{~dB}$ in dense multipath conditions.

Finally, through the analysis of the waist to head onbody channel, it was found that both first and second order statistics could be significantly improved using spatial diversity. Overall, these results suggest that implementing antenna diversity for the mitigation of on-body channel fading will also provide benefits for physical layer communications in mobileuser BAN applications, particularly in multipath environments. The work presented shows that careful consideration of both antennas and propagation can make a significant contribution in the realization of practical wearable systems for BAN. The achievable additional gain margin at the physical layer can be utilized in many ways such as improvements in link reliability, extended range, or directly to reduce the power consumption of BAN nodes. 
TABLE II

SUMMARY OF DIVERSITY STATISTICS FOR ALL BODYWORN DIVERSITY RECEIVER POSITIONS

\begin{tabular}{|c|c|c|c|c|c|c|c|c|c|c|c|c|}
\hline Antenna & \multicolumn{12}{|c|}{ Printed-F } \\
\hline Environment & \multicolumn{6}{|c|}{ Anechoic Chamber } & \multicolumn{6}{|c|}{ Reverberation Chamber } \\
\hline $\begin{array}{c}\text { Diversity } \\
\text { RX Position }\end{array}$ & $\rho_{c}$ & $\begin{array}{c}\mathrm{SC} \\
\text { (dB) }\end{array}$ & $\begin{array}{l}\text { EGC } \\
(\mathrm{dB})\end{array}$ & $\begin{array}{c}\text { MRC } \\
(\mathrm{dB})\end{array}$ & $\begin{array}{c}\text { DIMSL } \\
(\mathrm{dB})\end{array}$ & $\begin{array}{l}\text { MDPL } \\
(\mathrm{dBm})\end{array}$ & $\rho_{c}$ & $\begin{array}{c}\mathrm{SC} \\
\text { (dB) }\end{array}$ & $\begin{array}{l}\text { EGC } \\
(\mathrm{dB})\end{array}$ & $\begin{array}{c}\text { MRC } \\
(\mathrm{dB})\end{array}$ & $\begin{array}{c}\text { DIMSL } \\
(\mathrm{dB})\end{array}$ & $\begin{array}{l}\text { MDPL } \\
(\mathrm{dBm})\end{array}$ \\
\hline Head & 0.73 & 2.25 & 3.18 & 3.71 & 5.26 & -64.7 & 0.08 & 5.78 & 6.46 & 7.09 & 0.64 & -53.3 \\
\hline Front chest & 0.59 & 7.07 & 8.00 & 8.52 & 0.88 & -66.1 & 0.13 & 4.59 & 5.29 & 5.91 & 1.78 & -55.8 \\
\hline Waist & 0.62 & 1.99 & 3.05 & 3.53 & 7.83 & -65.7 & 0.61 & 3.95 & 4.90 & 5.39 & 3.82 & -49.5 \\
\hline Knee & 0.89 & 2.66 & 3.30 & 3.87 & 9.19 & -54.0 & 0.14 & 4.86 & 5.71 & 6.27 & 0.92 & -55.2 \\
\hline Ankle & 0.56 & 3.28 & 4.12 & 4.66 & 4.53 & -68.2 & 0.30 & 4.81 & 5.70 & 6.26 & 1.55 & -53.1 \\
\hline Wrist & 0.20 & 4.04 & 5.00 & 5.49 & 2.59 & -64.3 & 0.09 & 4.14 & 5.05 & 5.63 & 2.37 & -53.8 \\
\hline Elbow & 0.37 & 3.13 & 4.14 & 4.66 & 4.8 & -60.2 & 0.09 & 5.02 & 5.84 & 6.39 & 0.03 & -52.2 \\
\hline Back chest & 0.62 & 0.94 & 1.80 & 2.36 & 5.89 & -65.7 & 0.16 & 4.09 & 5.06 & 5.60 & 2.06 & -53.4 \\
\hline Antenna & \multicolumn{12}{|c|}{ WIA } \\
\hline Environment & \multicolumn{8}{|c|}{ Anechoic Chamber } & \multicolumn{4}{|c|}{ Reverberation Chamber } \\
\hline $\begin{array}{c}\text { Diversity } \\
\text { RX Position }\end{array}$ & $\rho_{c}$ & $\begin{array}{c}\mathrm{SC} \\
\text { (dB) }\end{array}$ & $\begin{array}{l}\text { EGC } \\
(\mathrm{dB})\end{array}$ & $\begin{array}{l}\text { MRC } \\
(\mathrm{dB})\end{array}$ & $\begin{array}{c}\text { DIMSL } \\
(\mathrm{dB})\end{array}$ & $\begin{array}{l}\text { MDPL } \\
(\mathrm{dBm})\end{array}$ & $\rho_{c}$ & $\begin{array}{c}\mathrm{SC} \\
(\mathrm{dB})\end{array}$ & $\begin{array}{l}\text { EGC } \\
(\mathrm{dB})\end{array}$ & $\begin{array}{c}\text { MRC } \\
(\mathrm{dB})\end{array}$ & $\begin{array}{c}\text { DIMSL } \\
(\mathrm{dB})\end{array}$ & $\begin{array}{l}\text { MDPL } \\
(\mathrm{dBm})\end{array}$ \\
\hline Head & 0.09 & 5.29 & 6.26 & 6.83 & 1.04 & -59.4 & 0.08 & 6.26 & 7.07 & 7.70 & 0.04 & -49.4 \\
\hline Front chest & 0.40 & 0.01 & 0.45 & 1.04 & 6.48 & -48.1 & 0.03 & 5.59 & 6.48 & 7.08 & 0.39 & -50.0 \\
\hline Waist & 0.35 & 1.31 & 2.84 & 3.18 & 3.29 & -65.6 & 0.06 & 5.76 & 6.56 & 7.20 & 0.05 & -49.3 \\
\hline Knee & 0.24 & 1.14 & 1.87 & 2.52 & 4.69 & -57.1 & 0.10 & 4.58 & 5.41 & 6.05 & 1.72 & -53.3 \\
\hline Ankle & 0.14 & 5.83 & 6.62 & 7.29 & 2.07 & -59.0 & 0.05 & 5.56 & 6.36 & 6.98 & 0.37 & -49.4 \\
\hline Wrist & 0.50 & 1.06 & 1.75 & 2.38 & 7.25 & -51.9 & 0.06 & 5.49 & 6.30 & 6.92 & 0.31 & -50.2 \\
\hline Elbow & 0.70 & 0.91 & 1.31 & 2.02 & 5.68 & -52.0 & 0.09 & 5.61 & 6.44 & 7.00 & 0.47 & -49.3 \\
\hline Back chest & 0.81 & 0.00 & 1.51 & 1.70 & 4.08 & -55.9 & 0.15 & 4.97 & 5.83 & 6.47 & 0.88 & -49.3 \\
\hline
\end{tabular}

\section{ACKNOWLEDGMENT}

The authors would like to thank Taconic Ltd, Tru-Lon Printed Circuits Ltd., Rohm GmbH (Rohacell foam) and Emerson and Cuming Ltd for product samples. We would also to acknowledge the assistance of Mr Jim Knox and the staff of the Queen's University engineering workshop.

\section{REFERENCES}

[1] S. Drude, "Requirements and application scenarios for body area networks," $16^{\text {th }}$ IEEE Mobile Wireless Comms. Summit, pp. 1-5, Jul. 2007.

[2] D. Puccinelli and M. Haenggi, "Wireless sensor networks: applications and challenges of ubiquitous sensing," IEEE Circuits Syst. Mag., vol. 5, 3, pp. 19-31, 2005.

[3] C. Otto, A. Milenkovic, C. Sanders, and E. Jovanov, "System architecture of a wireless body area sensor network for ubiquitous health monitoring," J. Mobile Multimedia, vol. 1, 4, pp. 307-326, 2006.

[4] E. Jovanov, A. Milenkovic, C. Otto, and P. C. de Groen, "A wireless body area network of intelligent motion sensors for computer assisted physical rehabilitation," J. Neuro Engineering \& Rehabilitation, vol. 2, 6, Mar. 2005

[5] J. Ryckaert, C. Desset, A. Fort, M. Badaroglu, V. de Heyn, P. Wambacq, et al., "Ultra-wide-band transmitter for low-power wireless body area networks: design and evaluation," IEEE Trans. Circuits Syst. I, vol. 52, 12, pp. 2515-2525, Dec. 2005.

[6] U. Varshney and S. Sneha, "Patient monitoring using ad hoc wireless networks: reliability and power management," IEEE Commun. Mag., vol. 44, 4, pp. 49-55 Apr. 2006.

[7] C. C. Y. Poon, Z. Yuan-Ting, and B. Shu-Di, "A novel biometrics method to secure wireless body area sensor networks for telemedicine and $\mathrm{m}$ health." IEEE Commun. Mag., vol. 44, 4, pp. 73-81, Apr. 2006.

[8] P. S. Hall, Y. Hao, Y. I. Nechayev, A. Alomainy, C .C. Constantinou, C. Parini, et al., "Antennas and propagation for on-body communication systems," IEEE Antennas Propagat. Mag., vol. 49, 3, pp. 41-58, Jun. 2007.

[9] G. A. Conway and W. G. Scanlon, "Antennas for over-the-body-surface communication at $2.45 \mathrm{GHz}$," IEEE Trans. Antennas Propagat., to appear.

[10] W. G. Scanlon and N. E. Evans, "Numerical analysis of bodyworn UHF antenna systems," IEE Electronics \& Communication Eng. Jnl., vol. 13, 2, pp. 53-64, 2001.

[11] M. Okoniewski and M. A. Stuchly, "A study of the handset antenna and human body interaction," IEEE Trans. Microwave Theory Tech., vol. 44, 10, pp. 1855-1864, Oct. 1996.
[12] K. L. Wong and C. I. Lin, "Characteristics of a 2.4-GHz compact shorted patch antenna in close proximity to a lossy medium," Microwave \& Optical Technology Ltrs., vol. 45, 6, pp. 480-483, 2005.

[13] S. L. Cotton and W. G. Scanlon, "Characterization and modeling of onbody spatial diversity within indoor environments at $868 \mathrm{MHz}$," IEEE Trans. Wireless Commun., to appear, 2008.

[14] A. A. Serra, P. Nepa, G. Manara, and P. S. Hall, "Diversity measurements for on-body communication systems," IEEE Antennas Wireless Propag. Lett., vol. 6, no. 1, pp. 361-363, 2007.

[15] S. L. Cotton and W. G. Scanlon, "Spatial diversity and correlation for off-body communications in indoor environments at $868 \mathrm{MHz}$," $65^{\text {th }}$ IEEE Vehicular Tech. Conf. VTC2007-Spring, pp. 372-376, Apr. 2007.

[16] D. Varshney, C. Arumugam, V. Vijayaraghavan, N. Vijay, and S. Srikanth, "Space-time codes in wireless communications," IEEE Potentials, vol. 22, 3, pp. 36-38, Aug. 2003.

[17] A. Hammoudeh and D. A. Scammell, "Frequency domain characterization of LOS non-fading indoor wireless LAN channel employing frequency and polarization diversity in the 63.4-65.4 GHz band," IEEE Trans. Veh. Technol., vol. 53, 4, pp. 1176-1189, Jul. 2004.

[18] L. C. Lukama, D. J. Edwards, and A. Wain, "Application of threebranch polarisation diversity in the indoor environment," IEE Proc. Communications, vol. 150, 5, pp. 399-403, 2003.

[19] J. Ryckaert, P. Doncker, R. Meys, A. de Le Hoye and S. Donnay, Channel model for wireless communication around human body, Electronic Letters, vol. 40, no. 9, pp. 543-544, April. 2004.

[20] IEEE Standards for Information Technology Part 15.1: Telecommunications and Information Exchange Between Systems, Local and Metropolitan Area Networks: Specific Requirements, Wireless Medium Access Control (MAC) and Physical Layer (PHY) Specifications for Low-rate WPAN, IEEE Std. 802.15.1-2002, 2002.

[21] IEEE Standards for Information Technology Part 15.4: Wireless Medium Access Control (MAC) and Physical Layer (PHY) Specifications for Low-Rate Wireless Personal Area Networks (LR-WPANs), IEEE Std. 802.15.4-2003, 2003.

[22] C. Gabriel, "4-Cole-Cole Analysis on compilation of the dielectric properties of body tissues at RF and microwave frequencies," Brooks Air Force Technical Report AL/OE-TR-1996-0037, 1996. Available (http://www.fcc.gov/fcc-bin/dielec.sh).

[23] S. L. Cotton and W. G. Scanlon "An experimental investigation into the influence of user state and environment on fading characteristics in wireless body area networks at $2.45 \mathrm{GHz}$," IEEE Trans. Wireless Commun., to appear, 2008.

[24] A. M. D. Turkmani, A. A. Arowojolu, P. A. Jefford, and C. J. Kellett, "An experimental evaluation of the performance of two-branch space and polarization diversity schemes at $1800 \mathrm{MHz}, "$ IEEE Trans. Veh. Technol., vol. 44, pp. 318-326, May 1995.

[25] C. B. Dietrich, K. Dietze, J. R. Nealy, W. L. Stutzman, "Spatial, 
polarization, and pattern diversity for wireless handheld terminals," IEEE Trans. Antennas Propagat., vol. 49, pp. 1271-1281, Sep. 2001

[26] W. C. Jakes, Microwave Mobile Communications. New York: Wiley, 1974.

[27] S. L. Cotton and W. G. Scanlon, "Characterization and modeling of the indoor radio channel at $868 \mathrm{MHz}$ for a mobile bodyworn wireless personal area network," IEEE Antennas Wireless Propag. Lett., vol. 6, pp. 51-55, 2007.

[28] M. D. Yacoub, C. R. C. M. da Silva, and J. E. Vargas Bautista, "Secondorder statistics for diversity-combining techniques in Nakagami-fading channels," IEEE Trans. Veh. Technol., vol. 50, pp. 1464-1470, Nov. 2001.

[29] C. D. Iskander and P. Takis Mathiopoulos, "Analytical level crossing rates and average fade durations for diversity techniques in Nakagami fading channels," IEEE Trans. Commun., vol. 50, pp. 1301-1309, Aug. 2002.

[30] E. Al-Hussaini and A. Al-Bassiouni, "Performance of MRC diversity systems for the detection of signals with Nakagami fading," IEEE Trans. Commun., vol. 33, pp. 1315-1319, Dec. 1985.

[31] S. O. Rice, "Statistical properties of a sine wave plus random noise," Bell Syst. Tech. J., vol. 27, pp. 109-157, Jan. 1948.

[32] N. C. Beaulieu and A. A. Abu-Dayya, "Analysis of equal gain diversity on Nakagami fading channels," IEEE Trans. Commun., vol. 39, pp. 225234, Feb. 1991.

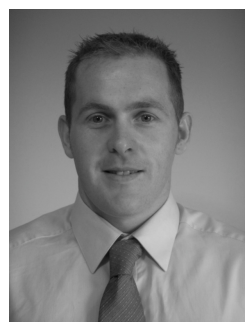

Gareth A. Conway was born in Armagh, UK, in 1981. He received the BEng Hons. Degree in Electronic Systems from the University of Ulster, UK in 2004. In 2005, he joined Queen's University of Belfast, (UK) where he is currently working towards a Ph.D. degree in Electronic Engineering. His current research interests include antennas, wave propagation and computational electromagnetism for wearable communications. Mr. Conway has authored or co-authored 13 international conference and journal papers since 2006. For his research on wearable on-body antennas, Mr. Conway was awarded the top prize in the SEMCAD-X global research competition recognizing outstanding research in the field of numerical simulation of electromagnetic fields in 2007.

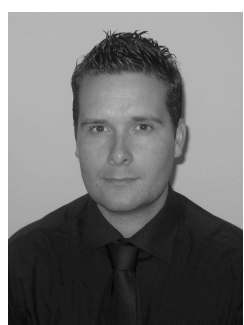

Simon L. Cotton (S'06-M'07) received the B.Eng. degree in electronics and software from the University of Ulster, UK in 2004 and the Ph.D. degree in electrical and electronic engineering from the Queen's University of Belfast, UK in 2007.

$\mathrm{He}$ is currently working as a Research Fellow with the Radio Communications Research Group of the Institute of Electronics, Communications and Information Technology, Belfast, UK, where he is investigating millimeter-wave technologies for personal communications and body-to-body networks. His research interests include radio channel characterization and modeling for wireless body and personal area networks, transceiver diversity in bodyworn applications and the simulation of wireless channels. Dr. Cotton has authored or co-authored over 16 international conference and journal papers since 2006.

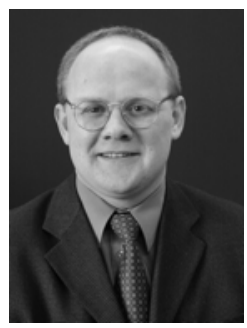

William G. Scanlon (M'97) received the B.Eng. degree in electrical engineering (first-class honours) by part-time study and the Ph.D. degree in electronics (specializing in wearable and implanted antennas) from the University of Ulster, UK in 1994 and 1997, respectively. He was appointed as Lecturer at the University of Ulster in 1998, then Senior Lecturer and Full Professor at Queen's University of Belfast (UK) in 2002 and 2008, respectively. He currently leads the Radio Communications research group at Queens. Prior to starting his academic career he had 10 years of industrial experience, having worked as a Senior RF Engineer for Nortel Networks, as a Project Engineer with Siemens and as a Lighting Engineer with GEC-Osram. Prof. Scanlon's current research interests include personal communications, wearable antennas, RF and microwave propagation, channel modeling and characterization, wireless networking and protocols and wireless networked control systems. He has published over 130 research papers in major IEEE/IET journals and in refereed international conferences. He served as a keynote speaker for the European Workshop on Conformal Antennas (2007). He will Co-Chair the 2009 Loughborough Antennas and Propagation Conference and he has acted as invited speaker and session chair at numerous other national and international conferences. Prof. Scanlon received a Young Scientist award from URSI in 1999, he is a prolific reviewer for IEEE/IET journals and conferences and other major conferences. He is a member of the IEEE International Committee on Electromagnetic Safety (ICES) and the IASTED International Committee on Telecommunications. 